\title{
Cirugía percutánea de la litiasis coraliforme ${ }^{*}$ Percutaneous nephrolithotomy for stag-horn lithiasis
}

\author{
Drs. OCTAVIO CASTILLO C. ${ }^{1,2}$, IVÁN PINTO G. ${ }^{1}$, MANUEL DÍAZ C. ${ }^{1}$, GONZALO VITAGLIANO ${ }^{1}$, \\ ALEJANDRO FONERÓN V. ${ }^{1}$, IVAR VIDAL M. ${ }^{1}$, RAFAEL SÁNCHEZ-SALAS ${ }^{1}$ \\ ${ }^{1}$ Unidad de Urología, Clínica Indisa, ${ }^{2}$ Departamento de Urología, \\ Facultad de Medicina, Universidad de Chile, Santiago, Chile.
}

\begin{abstract}
RESUMEN
Introducción: La litiasis coraliforme es una patología que de no ser tratada efectivamente lleva al daño renal progresivo e irreversible, con consecuencias que pueden ser letales. El abordaje clásico con cirugía abierta, aunque efectivo en la remoción del cálculo y sus fragmentos, está asociado a morbilidad importante. En las últimas décadas se han impuesto técnicas menos invasivas como la litotripsia extracorpórea, que tiene baja morbilidad pero un rendimiento discutible como monoterapia en cálculos complejos del riñón. Presentamos nuestra experiencia en nefrolitectomía percutánea de la litiasis coraliforme, técnica que conjuga una alta efectividad con baja morbilidad. Material y Método: Se analizan en forma retrospectiva los resultados de 42 pacientes portadores de litiasis coraliforme sometidos a nefrolitectomía percutánea como tratamiento único o combinado con litotripsia extracorpórea. Los pacientes se trataron en decúbito prono, con nefroscopio rígido, litotripsia endoscópica, con uno o más accesos en una o más sesiones. Todos fueron sometidos a tratamiento antibiótico previo. Entre los pacientes hubo un niño de 11 años, un paciente monorreno y una litiasis coraliforme en un riñón en herradura. Resultados: Se trataron 22 mujeres y 20 hombres de una edad promedio de 40,7 años (rango 11-74). En 27 casos el cálculo era derecho y en 15 izquierdo. En 33 casos (78,5\%) se realizó un acceso único, en 9 casos $(21,4 \%)$ se realizó 2 accesos y en 3 casos $(7,1 \%)$ se necesitó de 3 accesos. En 9 casos hubo litiasis residual que se trató con litotripsia extracorpórea y en 1 con ureteroscopía. En 40 pacientes se logró finalmente la remoción completa del cálculo y sus fragmentos $(95,3 \%)$. En la serie no hubo pérdida de unidades renales ni mortalidad. Conclusiones: La cirugía percutánea de la litiasis renal es una técnica mínimamente invasiva efectiva en el tratamiento de la litiasis coraliforme asociada a una baja morbilidad.
\end{abstract}

\section{PALABRAS CLAVE: Nefrolitotomía percutánea, litiasis coraliforme.}

\begin{abstract}
Background: Stag horn calculi may cause irreversible renal damage. Percutaneous nephrolithotomy is a minimally invasive surgical therapy than can be useful for these calculi. Aim: To report the experience with percutaneous nephrolithotomy for stag horn calculi. Material and Methods: Retrospective analysis of
\end{abstract}

\footnotetext{
* Recibido el 29 de Enero de 2008 y aceptado para publicación el 5 de Marzo del 2008.

Correspondencia: Dr. Octavio Castillo C.

Av. Apoquindo 3990, Of. 809, Santiago, Chile. Fax: (56-2) 2282524.

E-mail: octaviocastillo@vtr.net
} 
medical records of patients with stag horn calculi treated with percutaneous nephrolithotomy alone or associated to extracorporeal lithotripsy. All patients were treated with a rigid nephroscope, with one or more accesses during one or more sessions. Results: Twenty two women and 20 men aged 11 to 74 years were treated. One patient had only one kidney and one calculus was located in a horse shoe shaped kidney. The calculus was located in the right side in 27 patients and in the left side in 15. A unique access was used in 33 cases $(79 \%)$, two accesses in nine (21\%) and three accesses in three patients $(7 \%)$. Nine patients had a residual lithiasis that required extracorporeal lithotripsy and one required an ureteroscopy. In 40 patients $(95 \%)$, the complete removal of the calculus and its fragments was achieved. No patient died or lost the affected kidney. Conclusions: Percutaneous nephrolithotomy is a safe and effective minimally invasive surgical treatment for stag horn calculi.

\section{KEY WORDS: Stag horn calculi, percutaneous nephrolithotomy, lithotripsy.}

\section{INTRODUCCIÓN}

La litiasis coraliforme sigue siendo una patología de difícil manejo que con frecuencia compromete la integridad y la función renal. Aunque no existe un consenso estricto respecto a la definición de litiasis coraliforme, normalmente se entiende por tal una litiasis que forma un molde de la vía urinaria en la pelvis renal y al menos en parte de uno de los grupos calicilares. Lo habitual es que estos cálculos estén compuestos de fosfato, amonio y magnesio (estruvita), producto de la degradación de la urea por parte de bacterias productoras de ureasa como el Proteus. Se asume que por lo general éstos son cálculos colonizados por estos gérmenes, no sólo en su superficie sino en su interior, de tal manera que no es posible erradicar la infección sino hasta la remoción completa del cálculo. Estos cálculos comprometen la función renal por un fenómeno obstructivo e infeccioso. Las consecuencias son la atrofia renal progresiva y la intercurrencia de infecciones como pionefrosis, abscesos y pielonefritis xantogranulomatosa ${ }^{1,2}$.

El manejo clásico de la litiasis coraliforme era la nefrolitectomía anatrófica y la pielolitotomía extendida de Gil Vernet. La nefrolitotomía implica además de la lumbotomía, isquemia renal, nefrotomía bivalva amplia, extracción del cálculo y aseo de los fragmentos. Este abordaje, aunque tiene una alta eficacia, está asociado a importante morbilidad, particularmente relacionada al acceso. En las últimas décadas el acceso percutáneo del riñón ha ofrecido claras ventajas frente a la técnica abierta, aunque los cálculos de gran volumen siguen siendo un problema que obliga con frecuencia a crear accesos múltiples, en una o más sesiones en pabellón. Con el advenimiento de la litotripsia extracorpórea (LEC) a principio de los ochentas, la nefrolitectomía percutánea fue desplazada en la mayoría de la cirugía litiásica renal, incluyendo los cálculos coraliformes. En los últimos años, habiéndose aclarado los beneficios y sobretodo las limitaciones de la LEC, la nefrolitectomía percutánea ha vuelto a jugar un rol principal en la cirugía de la litiasis renal ${ }^{2}$. Presentamos a continuación nuestra experiencia en nefrolitectomía percutánea de la litiasis coraliforme.

\section{MATERIAL Y MÉTODO}

Se analizan en forma retrospectiva todos los pacientes portadores de una litiasis coraliforme, tratados por cirugía percutánea, en el período entre Abril de 1991 y Mayo de 2005. La serie está formada por 42 pacientes de una serie global de cirugías percutáneas de 231 casos tratados en el mismo período, lo cual corresponde al $18,2 \%$ de los casos. La técnica es realizada exclusivamente por urólogos, desde la punción renal hasta la extracción del cálculo.

La técnica quirúrgica ha sido ya descrita ${ }^{3}$, pero consiste básicamente en la instalación de un catéter ureteral con balón de oclusión (Microvasive ${ }^{\circledR}$ ) el cual permite contrastar la pelvis para la punción y al mismo tiempo evita la migración de fragmentos al uréter. Ya sea en decúbito ventral o dorsal preferimos la punción del cáliz inferior con aguja 18 G, la colocación de una guía Amplatz (Microvasive®) y la dilatación de trayecto con los dilatadores telescópicos de Alken. Se coloca una camisa de Amplatz 28 ó $30 \mathrm{Fr}$ y se comienza por la litotricia del cálculo localizado en cáliz inferior, continuando con la pelvis y los cálices accesorios, dejando para el final la limpieza de la unión pieloureteral. Si es necesario utilizar un segundo trayecto, colocamos una sonda Foley con el balón inflado en el Amplatz para contrastar la pelvis sin perder el medio de contraste. Para la fragmentación de los cálculos se utiliza un litotriptor neumático endoscópico.

En la preparación preoperatoria es fundamental un tratamiento antibiótico previo de 15 días de 
acuerdo a la sensibilidad del gérmen si éste se ha aislado, o con Ciprofloxacino en forma empírica, en dosis de $1 \mathrm{gr}$ al día.

Siempre dejamos una nefrostomía en el trayecto o en uno de los trayectos, si hubo más de 1 acceso, para una eventual re-entrada, la cual hacemos nunca antes de 48 horas.

\section{RESULTADOS}

La serie está formada por 42 pacientes, 22 mujeres y 20 hombres, con un promedio de edad de 40,7 años y un rango de 11 a 74 años. Veintisiete litiasis eran derechas y 15 eran izquierdas. Hubo 2 casos de obesidad mórbida, 1 paciente monorreno, 1 paciente con riñón en herradura y 1 paciente era portador del virus de Inmunodeficiencia adqui-

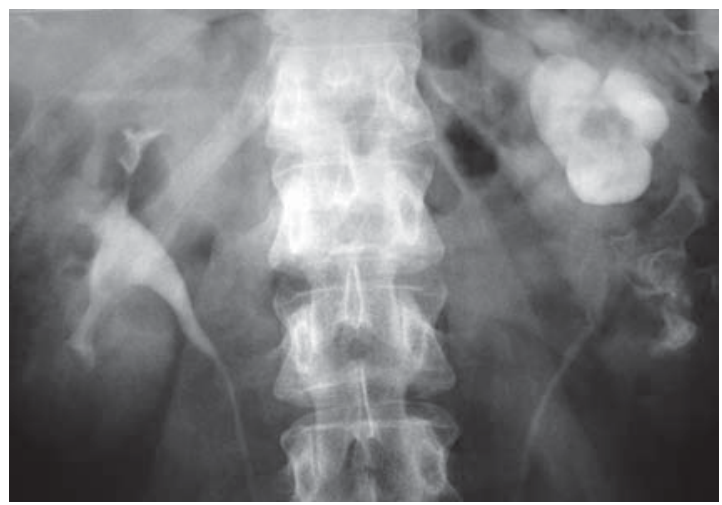

Figura 1. Pielografía de eliminación que muestra litiasis coraliforme parcial que ocupa pelvis y cálices inferiores del riñón izquierdo.

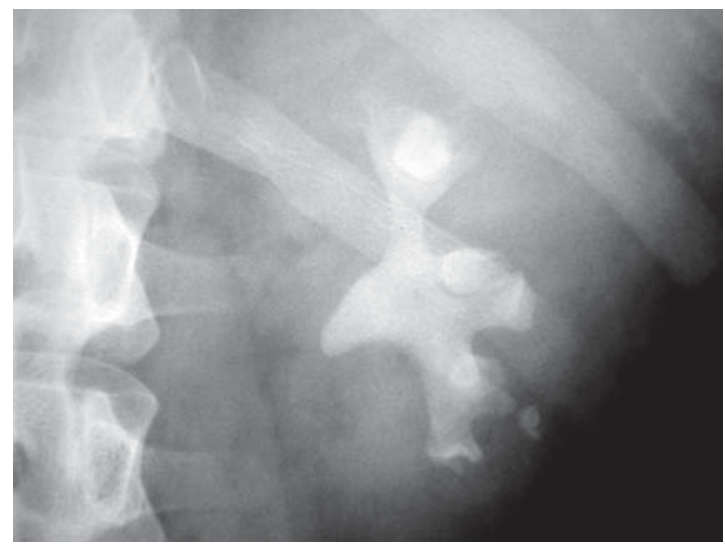

Figura 2. Radiografía no contrastada que muestra litiasis coraliforme que dibuja completamente las cavidades renales. rida. Un $50 \%$ de los pacientes tenía una o más sesiones de Litotripsia Extracorpórea en otros centros como monoterapia primaria o con la intención de hacer terapia combinada. La litiasis coraliforme fue calificada como coraliforme parcial si ocupaba toda la pelvis y al menos un cáliz (Figura 1) y coraliforme total si ocupaba la totalidad de la pelvis y cálices (Figura 2).

Por tratarse de un estudio retrospectivo no nos fue posible evaluar el volumen litiásico. La litiasis fue total en 25 pacientes $(59,5 \%)$ y parcial en 17 pacientes $(40,5 \%)$. El número de accesos fue de 1 en 33 casos (78,5\%), 2 accesos en $9(21,4 \%)$ y 3 accesos en 3 pacientes (7,1\%) (Figura 3$)$. El número de sesiones fue de 1 sesión en 31 pacientes $(73,8 \%), 2$ sesiones en $9(21,4 \%)$ y 3 sesiones en 1 caso $(2,4 \%)$ (Figura 4$)$.

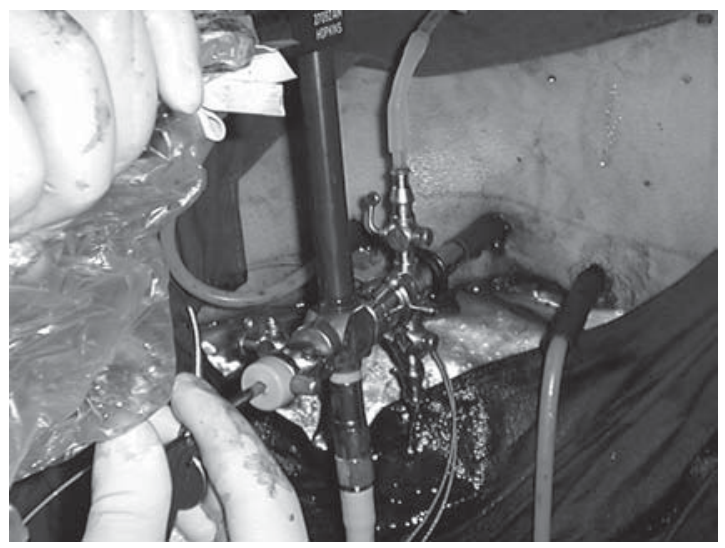

Figura 3. Se aprecian 3 accesos y el nefroscopio introducido por el acceso más central.

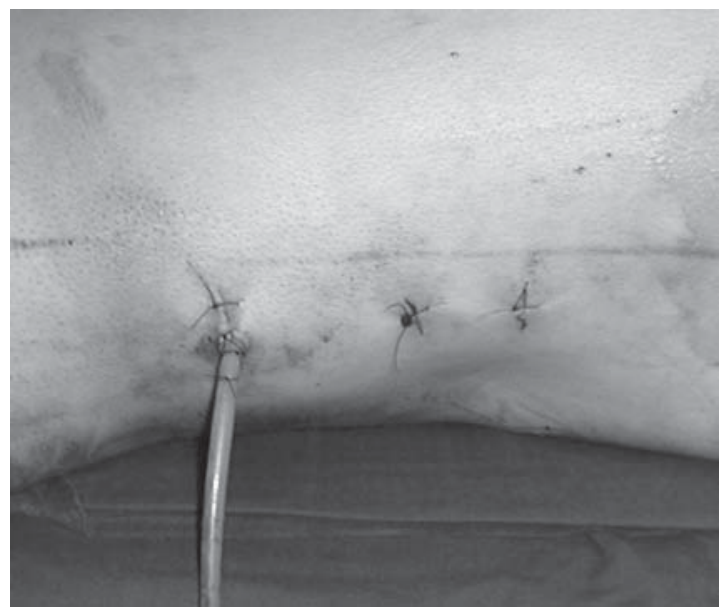

Figura 4. Nefrostomía por sólo uno de los accesos en un caso con accesos múltiples. Esta nefrostomía permite la re-entrada en caso de litiasis residual. 
Complicaciones ocurrieron en 5 pacientes $(11,9 \%)$ y fueron: Sepsis urinaria en 2 casos, extravasación de irrigante en 1 caso, sangrado (definido como la necesidad de transfundir glóbulos rojos) en 1 caso y calle litiásica en 1 caso, todos tratados con éxito. Litiasis residual hubo en 15 pacientes $(35,7 \%)$, las cuales fueron solucionadas con LEC en 9 casos, nueva sesión percutánea en 5 y ureteroscopia en 1 caso. La litiasis residual final fue de 2 casos $(4,7 \%)$.

No hubo casos de lesión colónica, pérdida de unidades renales ni mortalidad.

\section{DISCUSIÓN}

Desde la incorporación del concepto de acceso renal percutáneo por Goodwin en $1955^{4}$, ha habido avances dramáticos en la complejidad de la cirugía mínimamente invasiva de la litiasis renal, particularmente facilitados por la introducción del nefroscopio y de la litotripsia endoscópica. En 1976 Fernstrom describió la primera nefrolitectomía percutánea ${ }^{5}$, pero su desarrollo y masificación se frenaron en algún grado frente a la popularidad que ganaron otras técnicas poco invasivas como la litotripsia extracorpórea y la ureteroscopía. En los últimos años, al haber crecido la evidencia respecto al rendimiento más bien bajo de la LEC en litiasis complejas del riñon, la nefrolitectomía percutánea parece retomar un lugar principal ${ }^{6}$. En un estudio de Morris y cols, donde analizan los datos de 12.948 pacientes sometidos a nefrolitectomía percutánea en un período de 4 años en Estados Unidos, demuestran que la frecuencia del uso de la técnica se duplicó en el período estudiado y que la mortalidad relacionada al procedimiento $(0,2 \%)$ es muy inferior en centros con gran volumen de cirugías ${ }^{7}$. En el último panel de la AUA para el manejo de la litiasis coraliforme, la recomendación es la nefrolitectomía percutánea como primera alternativa. A diferencia del reporte del mismo panel en 1994, los metanálisis muestran mejor rendimiento (estado libre de cálculos) con la técnica percutánea como monoterapia que en su combinación con LEC².

A la fecha sólo existe un estudio prospectivo randomizado que compara la nefrolitectomía percutánea con cirugía anatrófica para litiasis coraliforme. En éste se demuestra una menor morbilidad asociada al procedimiento endoscópico y recuperación más rápida, a la vez que ambas tienen un rendimiento similar en cuanto a porcentaje libre de cálculos $(74 \%$ y $82 \%$ para cirugía percutánea y abierta respectivamente $)^{8}$.

En nuestra serie hay un caso de litiasis coraliforme en un riñón en herradura tratado exitosa- mente con cirugía percutánea. Éstos representan casos de gran dificultad debido a las anomalías anatómicas. Con frecuencia en estos pacientes se requieren accesos en los cálices superiores, lo que aumenta el riesgo de lesión pleural. Existen series con altísimas tasas de estado libre de cálculos en riñones en herradura con nefroscopios flexibles ${ }^{9}$.

La baja frecuencia de litiasis coraliforme en niños no permite establecer guías de tratamiento. Existen numerosas series de LEC en este grupo de pacientes, con buenos resultados especialmente desde el punto de vista de la baja morbilidad ${ }^{10}$. En nuestra serie hay un niño de 11 años tratado exitosamente con cirugía percutánea quien no presentó complicaciones.

En nuestros pacientes dejamos de rutina una nefrostomía con una sonda Foley $20 \mathrm{~F}$. Aunque no hemos tenido problemas relacionados a la presencia de la nefrostomía, existen trabajos que sugieren que catéteres más finos o eventualmente el uso de sellantes en el trayecto (sin nefrostomía) permiten un postoperatorio con menos dolor cuando la remoción del cálculo ha sido completa ${ }^{5,11}$.

Un estudio publicado recientemente demuestra que el acceso percutáneo realizado por urólogos (sólo $11 \%$ en Estados Unidos) está asociado a mejor rendimiento y menores complicaciones que los realizados por radiólogos intervencionistas ${ }^{12}$.

Finalmente, nuestra serie muestra resultados comparables a los metanálisis efectuados por el último panel en litiasis coraliforme de la Asociación Americana de Urología².

\section{CONCLUSIONES}

La nefrolitectomía percutánea de la litiasis coraliforme es un procedimiento mínimamente invasivo que tiene buen rendimiento comparado con otras técnicas de nefrolitectomía o litotripsia, que se asocia a baja morbilidad, particularmente cuando es efectuado por urólogos entrenados.

\section{REFERENCIAS}

1. Lingeman J, Lifshitz D, Evan A. Surgical Management of Urinary Lithiasis. En: Campbell's Urology. Philadelphia: Editorial Saunders, 2002; 3361-3451.

2. Preminger G, Assimos D, Lingeman J, Nakada S, Pearle M, Wolf J. Chapter 1: AUA Guideline in Management of Staghorn Calculi: Diagnosis and Treatment Recommendations. J Urol 2005; 173: 1991-2000.

3. Castillo O, Van Cauwelaert R, Aguirre C. Cirugía percutánea de la litiasis renal: análisis de 224 pacientes. Rev Chil Cir 1991; 43: 150-154. 
4. Goodwin WE, Casey WC, Woolfe W. Percutaneous trocar (needle) nephrostomy in hydronephrosis. JAMA 1955; 157: 891-894.

5. Fernstrom I, Johansson B. Percutaneous Pyelolithotomy. A New Extraction Technique. Scand J Urol Nephrol 1976; 10: 257-259.

6. Kim S, Kuo R, Lingeman J. Percutaneous Nephrolithotomy: An Update. Curr Opin Urol 2003; 13: 235-241.

7. Morris D, Wei J, Taub D, Dunn R, Wolf J, Hollenbeck B. Temporal Trends in the Use of Percutaneous Nephrolithotomy. J Urol 2006; 175: 1731-1736.

8. Al-Kohlany K, Shokeir A, Mosbah A, Mohsen T, Shoma A, Eraky I, et al. Treatment of Complete Staghorn Stones: A Prospective Randomized Comparison of Open Surgery Versus Percutaneous
Nephrolithotomy. J Urol 2005; 173: 469-473.

9. Raj G, Auge B, Weizer A, Denstedt J, Watterson J, Beiko D, et al. Percutaneous Management of Calculi Within Horseshoe Kidneys. J Urol 2003; 170: 48-51.

10. Lottman H, Traxer O, Archambaud F, MercierPageyral B. Monotherapy Extracorporeal Shock Wave Lithotripsy for the Treatment of Staghorn Calculi in Children. J Urol 2001; 165: 2324-2327.

11. Pietrow $P$, Auge B, Lallas $C$, Santa-Cruz R, Newman G, Albala D, et al. Pain After Percutaneous Nephrolithotomy: Impact of Nephrostomy Tube Size. J Endourol 2003; 17: 411-414.

12. Watterson J, Soon Sh, Jana K. Access Related Complications During Percutaneous Nephrolithotomy: Urology Versus Radiology at a Single Academic Institution. J Urol 2006; 176: 142-145. 